\title{
Baldermath: A Design-Based Research Approach to Developing a Game for Student Work
}

\section{Analysis}

Theoretical Framework: To become professionally accomplished, teachers must both develop pedagogical content knowledge as well as come to engage in ambitious practices (Ball \& Cohen, 1999). Prior research has identified student work analysis as a promising task for use with prospective and in-service teachers for developing both of these capacities (Windschitl, et al. 2011; Kazemi \& Franke, 2003).

However, not all looking at student work (LASW) helps to develop productive practices, (Ball \& Cohen, 1999; Little, 2003). To facilitate learning, teacher educators and leaders of professional development have developed protocols for use in LASW sessions (e.g. Looking At Student Work, 2000; Dearman \& Alber, 2005). These protocols serve to undercut teachers’ instinct to immediately evaluate the quality of student work, and they structure participation and organize opportunities for different modes of engagement with student work (such as open-ended noticing and raising questions) (Little, 2003). Protocols -- or facilitation that similarly structures interactions in the group -- are an important element of LASW.

High-quality engagement in LASW for mathematics teachers, in particular, can lead to teachers paying increased attention to the details of student thinking in their classrooms (Kazemi \& Franke, 2003). In case studies of practice, the role of the facilitator in workgroups went beyond ensuring adherence to a protocol. For example, facilitators can consistently press participants to attend more closely to the details of student work, since teachers at first think that student work “speaks for itself” and does not demand inquiry (Kazemi \& Franke, 2003). This makes high-quality LASW a facilitator-intensive learning activity. In addition, LASW typically 
involves participants bringing in student work from their own classrooms (Little, 2003). These two factors -- the importance of the facilitator and the need for participants to supply the student work -- severely limit the opportunities available to prospective mathematics teachers to learn to attend more closely to student thinking through a LASW experience.

Research Questions: In this study, we use design-based research methods to develop a game to aid instructors in creating high-quality LASW opportunities for prospective teachers. Games have the potential to provide a situated, action-oriented, embodied experience where teachers can take the "projective identity" of students or other teachers (Gee, 2003). In addition, games can trigger emotional and psychological responses that spark empathy and perspective taking (Boltz et al., 2015). Actions and choices that players make in the game are "pleasantly frustrating" and playful (Gee, 2003). This embodied experience within a game can help teachers to develop empathy for students' perspective and approximate desirable practices around LASW.

The rules of a game play a role similar to that of protocols in structuring LASW. Given the ability of games to create experiences of simulation and role-taking, we further hypothesized that gameplay could encourage prospective teachers to attend more carefully to details in student work, even using student work not brought in by the participants themselves and in the absence of strong facilitation.

To investigate how games can be used as a context to facilitate high quality LASW, we developed a game:Baldermath. Baldermath asks players to create responses with mistakes to mathematics questions that resemble work that a student could produce. One player has been assigned a genuine piece of student work, and this player recreates this student work in their own hand. The judge's role is to guess which piece of player-generated work was based off of genuine 
student work. Success in the game occurs when the judge correctly identifies the genuine student-work, or when a player generates student work that successfully passes for the work of an actual student.

Using a design-based research (DBR) approach to our research (Easterday, Rees, Gerber 2014), we follow a process of researching learner needs, rapidly prototyping learning experiences to meet those needs, testing prototypes with learners, and iteratively revising our prototypes and tests. Our research aims to address an overarching question: Given that a well-facilitated LASW experience can lead to productive shifts in teacher knowledge and practice, can contextualizing LASW in a game yield similar benefits while reducing the facilitation load? In this presentation, we report our first inquiry cycle and iteration focusing on the question of whether the game mechanics of Baldermath structure LASW in a way similar to existing protocols, as this is a minimal condition for yielding the benefits of LASW (Little, 2003).

Methodology: Design of the Game - A review of the literature lead to our identifying key features of LASW that our game would aim to instantiate: (1) talk focuses on the student work at hand; (2) participants are encouraged to attend to details present in the student work; (3) each participant has an opportunity to make sense of student thinking.

We then gathered in-service teachers, teacher educators, and game design experts for a collaborative design session. We presented the key features of LASW and asked the group to develop rough-draft prototypes of a game that instantiated these key features. Our aim for this design session was to quickly uncover the terrain of LASW-game possibilities. 
Analysis of the collaborative design session yielded a most promising prototype.

Designers connected the careful attention that we sought to foster in prospective teachers with the deliberation needed to decide whether a truth or falsehood has been told in games such as Balderdash and Wait, Wait, Don't Tell Me... This lead to our prototyping a game on the model of these truth-telling/bluffing games.

Participants and Setting With a prototype, we proceeded to test the game. Testing proceeded in four sessions over two months, with a total of seven sessions of Baldermath with 3-7 players each. Participants included novice teachers, teacher educators, and mid-career teachers, recruited as a convenience sample from teacher networks cultivated by the authors. Three of these testing sessions were in-person; one was performed online via video chat. After examining data from each playtest round, we made improvements to the core gameplay and our evaluation protocols. We report below a synthesis of processes and results across these sessions.

Sources of Data Game testing sessions proceeded in three stages. First, groups were given a piece of student work and asked to take turns responding to an open-ended prompt about the student work (“Take turns sharing your thoughts about the student work.”). After each participant had shared thoughts, the game was introduced and played. Finally, testing sessions closed with an open-ended survey that encouraged participants to reflect on the session (e.g. "How would you describe the intellectual work you were doing while playing the game?”).

We collected recordings of testing sessions and in-game artifacts created during the game. To improve the quality of the game, we used our ongoing collection of data to revise gameplay. For instance, in early versions of the game participants included written rationales of the work they produced. When transcript analysis and survey feedback indicated that enjoyment and 
engagement with the game was reduced due to the time needed to generate responses to the mathematics problem, the written rationale was eliminated.

We did not seek evidence of changes in teacher practice; the evidence we gathered was oriented towards "proof-of-concept," where we sought to confirm that the game we had developed was sufficiently promising to justify further research. We conceptualized “proof-of-concept” to constitute passing two thresholds of evidence: (1) evidence that the game functions to restrict teacher attention to the student work (thus serving a similar function to protocols for LASW) and (2) evidence that the game functions to draw attention to details in student work that they otherwise would not have attended to (thus serving a similar function to facilitators in high-quality mathematics LASW sessions). Our analysis aimed to show that Baldermath passed this threshold and thus would benefit from further study.

\section{Summary of Findings}

When analyzing session recordings, we sought to compare participant talk in response to student work with an open-ended prompt to teacher talk about student work in the context of Baldermath. Our analysis was oriented towards addressing our two proof-of-concept questions. Analysis of recording transcripts indicated that Baldermath functions to restrict teacher talk and encourages teachers to meaningfully interpret student work.

Restricting Talk to Student Work: Previous research notes ways in which conversations surrounding student work can drift away from the work itself into other issues relating to students and instruction (Little, 2003). When asked to respond to student work with an open-ended prompt, participants display a similar capacity to drift away from the student work itself. For example, one participant -- Kent, a mid-career middle-school mathematics teacher -- 
shifted conversation from the student work to speculation about the instructional causes of the mistake:

They had that extra eighth flying around that they decomposed and they don't know how to add it to three-fourths properly so they... This seems like a potentially an artifact of instruction. Like maybe the student incorporating something like a strategy that they've seen in a different context but doesn't know how to apply it.

Later in the session, Kent was tasked with making sense of another student mistake as part of gameplay. During his turn, he described student thinking without reference to possible instructional choices, characteristics of the student or other possibilities. When reflecting on his experience after the game had ended, Kent describes feeling compelled by gameplay to ascribe meaning to an element of the student work that he did not see as meaningful:

Yeah, I don't know. The interesting thing was, there wasn't a clear answer... I didn't see the connection for two-fifths and three-tenths, so I had to just make up the skipping around thing. Like, I saw how they answered three of the four questions, but it wasn't clear to me... There wasn't any work that I could see that indicated three-tenths anywhere. Whereas Kent had earlier shown a willingness to relate aspects of student work to prior instruction, he expresses a feeling of being pressed to offer an explanation that focused on the student work. This supports our hypothesis that Baldermath can apply pressure on participants to restrict their talk to the student work, fulfilling the first of our "proof-of-concept" conditions. Encouraging Meaning-Making of Student Mistakes: Kent's explanation of the confusing aspects of the student work not only were restricted to the student work, but positioned the student as an expression of meaningful mathematical thought (as opposed to e.g. a "stupid mistake”). 
And then, I saw that there was sort of a connection between the next two, you were comparing two-fifths to three-tenths and then two-fifths to three-sevenths. So I did two-fifths to three-sevenths first and I drew that out and I did it with bars, instead of circles 'cause it's hard for me to cut circles into seven pieces or five pieces. And I saw that two-fifths was bigger than three-sevenths. So then I figured three-tenths is smaller than three-sevenths, so there's no way it would be bigger than two-fifths. So then I just split two-fifths for both of them.

This sharply contrasts with this participant's explanation of the student work before the game, where he situates the student's work as non-meaningful (i.e. as an artifact of instruction). This constitutes a devotion of attention towards the details of student work.

Significance: The results of our work suggest that Baldermath is a promising context for LASW deserving of further study. In this investigation we did not aim to collect data supporting the efficacy of Baldermath in leading to the shifts in practice that high-quality LASW is capable of. Further research should investigate these questions of efficacy over a longer investigation.

LASW is theorized to be effective as an approximation of practice -- simulations of the complex interactions constitutive of teaching (Grossman, 2009). Could games constitute such approximations of practice? On one hand, roleplay and simulation are inherently playful activities, and therefore games could be a natural setting for approximations of practice in teacher education. On the other hand, games, by design, introduce artificiality. This artificiality raises the possibility that games lack face-similarity with teaching, and learning grounded in a game is unable to be deployed in practice. 
Our work suggests that Baldermath could be able to capture core elements of a

high-quality approximation of practice -- LASW. Further research into Baldermath will inform our understanding of the role of games in professional education more broadly.

\section{References:}

Ball, D. L., \& Cohen, D. K. (1999). Developing practice, developing practitioners: Toward a practice-based theory of professional education.Teaching as the learning profession: Handbook of policy and practice, 1, 3-22.

Boltz, L. O., Henriksen, D., \& Mishra, P. (2014). Rethinking technology \& creativity in the 21st century: Empathy through gaming-perspective taking in a complex world. TechTrends, 59(6), 3-8.

Dearman, C. C., \& Alber, S. R. (2005). The changing face of education: Teachers cope with challenges through collaboration and reflective study. The Reading Teacher, 58(7), 634-640.

Easterday, M., Rees Lewis, D., \& Gerber, E. (2014). Design-based research process: Problems, phases, and applications. In Proc. of International Conference of Learning Sciences (Vol. 14).

Gee, J. P. (2003). What video games have to teach us about learning and literacy. Computers in Entertainment (CIE), 1(1), 20-20.

Grossman, P., Compton, C., Igra, D., Ronfeldt, M., Shahan, E., \& Williamson, P. (2009). Teaching practice: A cross-professional perspective. Teachers College Record, 111(9), 2055-2100.

Kazemi, E., \& Franke, M. L. (2004). Teacher learning in mathematics: Using student work to promote collective inquiry. Journal of mathematics teacher education, 7(3), 203-235.

Little, J. W., Gearhart, M., Curry, M., \& Kafka, J. (2003). Looking at student work for teacher learning, teacher community, and school reform. Phi Delta Kappan, 85(3), 185.

Looking at Student Work. (2000). Retrieved September 02, 2016, from http://www.lasw.org/

Windschitl, M., Thompson, J., \& Braaten, M. (2011). Ambitious pedagogy by novice teachers: Who benefits from tool-supported collaborative inquiry into practice and why. Teachers College Record, 113(7), 1311-1360. 